\title{
Preclinical evaluation of gold-DTDTPA nanoparticles as theranostic agents in prostate cancer radiotherapy
}

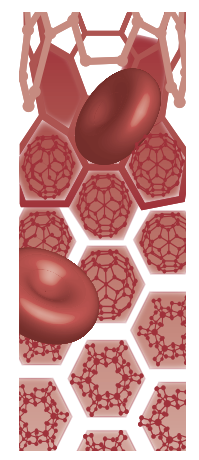

\begin{abstract}
Aim: Gold nanoparticles have attracted significant interest in cancer diagnosis and treatment. Herein, we evaluated the theranostic potential of dithiolated diethylenetriamine pentaacetic acid (DTDTPA) conjugated AuNPs (Au@DTDTPA) for CT-contrast enhancement and radiosensitization in prostate cancer. Materials \& methods: In vitro assays determined Au@DTDTPA uptake, cytotoxicity, radiosensitizing potential and DNA damage profiles. Human PC3 xenograft tumor models were used to determine $\mathrm{CT}$ enhancement and radiation modulating effects in vivo. Results: Cells exposed to nanoparticles and radiation observed significant additional reduction in survival compared with radiation only. Au@DTDTPA produced a CT enhancement of $10 \%$ and a significant extension in tumor growth delay from 16.9 days to 38.3 compared with radiation only. Conclusion: This study demonstrates the potential of Au@DTDTPA to enhance CT-image contrast and simultaneously increases the radiosensitivity of prostate tumors.
\end{abstract}

First draft submitted: 19 February 2016; Accepted for publication: 5 May 2016; Published online: 27 July 2016

Keywords: cancer $\bullet$ CT enhancement $\bullet$ DTDTPA $\bullet$ gold nanoparticles $\bullet$ prostate $\bullet$ radiation enhancement $\bullet$ SARRP

The unique properties of nanoparticles offer significant potential for the development of novel materials that provide innovative solutions to both diagnostic and therapeutic challenges in medicine. Nanoscale theranostic approaches, integrating properties for cancer diagnosis and therapy, could offer significant advantages over separately administered diagnostic and therapeutic agents [1-3]. Where a single nanoparticle formulation may be limited to targeting a specific biological process, the abilities of multifunctional nanoparticles to simultaneously enhance imaging and therapy could offer much improvement beyond current standard of care.

Gold nanoparticles (AuNPs) are among the most widely studied nanoagents, largely due to a variety of unique physical, chemical and optical properties. Notable features include ease of synthesis, relative biocompatibility, tune- able optical properties based on the surface plasmon resonance (SPR; associated with size and surface geometry of the nanoparticle) and importantly the ability to manipulate downstream applications through the conjugation of secondary functional groups [4-11]. Exploiting the electron dense nature of gold has led to the evaluation of AuNPs as contrast agents for a wide range of imaging modalities including optical, x-ray, computed tomography (CT), Raman spectroscopy, multiphoton microscopy and photo-acoustic imaging [12-16].

In addition, AuNPs have been well documented as radiosensitizers in in vitro and in vivo models (reviewed in Butterworth et al.) [17,18], with effects attributed to a combination of physical (secondary electron liberation), chemical (radiolysis mediated effects) and biological (DNA damage/reactive oxygen species [ROS] scavenging responses) effects [18-20].
Karl T Butterworth ${ }^{\dagger, 1}$, James R Nicol $^{\dagger, 2}$, Mihaela Ghita', Soraia Rosa', Pankaj Chaudhary', Conor K McGarry', Helen O McCarthy², Gloria JimenezSanchez ${ }^{3}$, Rana Bazzi ${ }^{3}$, Stéphane Roux ${ }^{3}$, Olivier Tillement ${ }^{4}$, Jonathan A Coulter ${ }^{*, \neq, 2}$ \& Kevin M Prise ${ }^{* *, \neq, 1}$ ${ }^{1}$ Centre for Cancer Research \& Cell Biology, Queen's University Belfast, 97 Lisburn Road, Belfast, BT9 7AE, UK ${ }^{2}$ School of Pharmacy, Queen's University Belfast, 97 Lisburn Road, Belfast, BT9 7AE, UK

'UTINAM Institute, UMR 6213 CNRS - UFC, University of Franche-Comté, Besançon 25030, France ${ }^{4}$ The Institute of Light and Matter, UMR 5306 CNRS - UCBL, University of Lyon, Villeurbanne 69622, France

*Author for correspondence: j.coulter@qub.ac.uk

**Author for correspondence:

k.prise@qub.ac.uk

${ }^{+}$Authors contributed equally to this work ₹Senior authorship 
CT imaging is frequently used to generate tomographic images of soft tissue and vasculature, allowing high levels of precision in differentiating tissue types, and aiding tumor diagnosis and treatment planning. AuNPs provide excellent contrast, owing to their electron dense nature and SPR. The superior absorption coefficient of gold relative to soft tissue, bone and tumor, generates up to three-times more contrast per unit weight compared with iodine [12]. Reuveni et al. functionalized AuNPs with anti-EGFR, which were injected into mice with human squamous cell carcinoma head and neck cancer, and subsequently imaged with CT. Untargeted and EGFR conjugated AuNPs significant enhanced tumor contrast by 2.3 - and 5.6-fold, respectively, over control levels [21].

Rationally, AuNPs should enhance passive tumor specific accumulation due to the vascular pathophysiology, referred to as the enhanced permeability and retention effect (EPR), first described by Maeda et al. [22]. To ensure colloidal stability in physiological conditions, diethylenetriaminepentacetic acid (DTPA) was grafted onto $2.4 \mathrm{~nm}$ AuNPs through two thiol moieties, termed dithiolated diethylenetriamine pentaacetic acid (DTDTPA), with the AuNP conjugate referred to as Au@DTDTPA [23]. In previous biodistribution studies these nanoparticles were shown to passively accumulate within tumor tissue, and are rapidly cleared without reticuloendothelial retention by renal excretion [24]. Furthermore, Au@DTDTPA conjugated with gadolinium (Au@DTDTPA-Gd ${ }_{50}$ ) demonstrated efficient renal clearance while simultaneously generating enhanced CT and MRI capability [24]. In a separate study, osteosarcoma bearing animals were treated with microbeam radiation therapy, improving median survival time from 42.5 days to 61 following the inclusion of Au@DTDTPA. At this point the study was terminated, with an apparent disappearance of the tumor in six of the seven animals, demonstrating a major survival improvement under microbeam radiation [25].

In the current study, we aimed to evaluate the theranostic potential of Au@DTDTPA nanoparticles in a series of cell-based assays and using a preclinical model of prostate cancer radiotherapy. To date, radiosensitizing studies of AuNPs in prostate cancer remain limited, with only one study demonstrating in vivo efficacy using goserelin functionalized gold nanorods [26]. In this study we report, for the first time, preclinical rationale for the use of Au@DTDTPA as an efficient theranostic agent in prostate cancer.

\section{Materials \& methods}

\section{Synthesis of Au@DTDTPA nanoparticles}

Au@DTDTPA nanoparticles were synthesized according to the protocol of Brust $e t a l$. [27] and as previously described by Debouttiere et al. [23]. $\mathrm{HAuCl}_{4} \cdot 3 \mathrm{H}_{2} \mathrm{O}$ was reduced with $\mathrm{NaBH}_{4}$ in the presence of DTDTPA, a thiol stabilizer which adsorbs onto the surface of the growing particles and ensures control of size and stability of the colloid. Au@DTDTPA nanoparticles were prepared at a concentration of $10 \mathrm{~g} / \mathrm{l}$ in a deionized water solution and subsequent dilution in cell culture media was performed for storage.

\section{Physical characterization of Au@DTDTPA nanoparticles}

The physical properties of Au@DTDTPA nanoparticles were characterized by transmission electron microscopy [24] and dynamic light scattering. High-resolution transmission electron microscopy (HRTEM) was carried out using a JEOL 2010 microscope operating at $200 \mathrm{kV}$. The hydrodynamic size, charge and polydispersity index (PDI) of Au@DTDTPA as synthesized and following incubation in medium containing $10 \%$ FBS and was measured using dynamic light scattering with a Malvern Zetasizer ZS (Malvern Instruments, Malvern, UK).

\section{Cell culture}

The human prostate cancer cell lines, PC3 and DU145, and the immortalized normal prostate epithelial cell line, PNT2-C2, were cultured in Roswell Park Memorial Institute medium (RPMI-1640) supplemented with $10 \%$ fetal calf serum (FCS) and 1\% penicillin-streptomycin. PC3 and DU145 cells were purchased from the American Type Culture Collection []. PNT2-C2 were kindly donated by Prof Norman Maitland at the University of York [28]. Cells were maintained in a humidified atmosphere at $37^{\circ} \mathrm{C}$ with $5 \% \mathrm{CO}_{2}$ and subcultured every $3-4$ days to maintain exponential growth.

\section{Atomic absorption spectroscopy}

Cells $\left(2.5 \times 10^{5}\right)$ were seeded, allowed to adhere for $24 \mathrm{~h}$ and incubated with media containing 100, 250 and $500 \mu \mathrm{g} / \mathrm{ml}$ Au@DTDTPA for a further $24 \mathrm{~h}$. AuNPs were then aspirated and cells washed twice with PBS to remove surface bound particles, trypsinized and centrifuged at $8000 \mathrm{rcf}$ for $5 \mathrm{~min}$. The supernatant was removed and the pellet resuspended in $1 \mathrm{ml} \mathrm{PBS}$. A $50 \mu \mathrm{l}$ aliquot of the cell suspension was removed and counted to determine the cell number. The remaining sample was dissolved in $1 \mathrm{ml}$ of aqua regia ( 1 part $70 \%$ nitric acid, to 3 parts $37 \%$ hydrochloric acid). Each sample was made up to $5 \mathrm{ml}$ with $18.2 \mathrm{M} \Omega \cdot \mathrm{cm}$ water and the gold content determined by atomic absorption spectroscopy (AAS) with flame atomizer to measure absolute quantification of elemental gold. 


\section{In vitro cytotoxicity}

The cytotoxicity of Au@DTDTPA nanoparticles was measured using the fluorescent Alamar blue assay. $1.5 \times 10^{4}$ cells were plated in a 96-well plate, allowed to adhere for $24 \mathrm{~h}$ then supplemented with 100,250 and $500 \mu \mathrm{g} / \mathrm{ml} \mathrm{Au@DTDTPA} \mathrm{for} \mathrm{a} \mathrm{further} 24 \mathrm{~h}$. Excess Au@DTDTPA were removed, the cells washed twice with PBS and cells incubated in media containing 10\% Alamar blue reagent for $4 \mathrm{~h}$. Media was then transferred to a 96-well black out plate and read on a fluorescent plate reader at $570 \mathrm{~nm}$ excitation and $585 \mathrm{~nm}$ emission.

\section{Clonogenic assay \& in vitro irradiation procedure}

A total of $2.5 \times 10^{5}$ exponentially growing cells were plated in $35 \mathrm{~mm}^{2}$ dishes and allowed to adhere overnight before incubation with Au@DTDTPA nanoparticles in complete culture medium for $24 \mathrm{~h}$. Excess nanoparticles were removed, cells washed twice with PBS and incubated in complete media prior to irradiation. In vitro experiments were performed with $225 \mathrm{kVp}$ x-rays generated using a X-Rad 225 generator (Precision X-ray Inc., CT, USA) with a $2 \mathrm{~mm}$ copper filter. All quoted doses are the absorbed dose in water $50 \mathrm{~cm}$ from the radiation source at a dose rate of $0.591 \mathrm{~Gy} / \mathrm{min}$. Following irradiation, cells were trypsinized, diluted $1: 1$ in fresh medium and vortexed to ensure a homogenous solution before being counted and reseeded at low densities for clonogenic assay as described by Puck and Marcus [29]. After 14 days, colonies were fixed with $0.4 \%$ crystal violet in $70 \%$ methanol and counted applying a 50-cell exclusion criterion. Surviving fraction was calculated as previously reported [30].

\section{DNA damage analysis by immunofluorescence microscopy}

Cells were plated at a density of $2 \times 10^{4}$ cells onto 4 chamber microscope slides and allowed to adhere overnight. A solution of $250 \mu \mathrm{g} / \mathrm{ml}$ of AuNP was added to the cells and incubated for $24 \mathrm{~h}$ prior to irradiation with 1 Gy. Subsequently, cells were fixed using a 50\% $\mathrm{MeOH} / 50 \%$ acetone solution at 1 or $24 \mathrm{~h}$ post treatment to determine if Au@DTDTPA enhanced double strand break damage or prolonged DNA damage repair. Cells were rinsed three-times with PBS (Sigma, UK) and incubated with a blocking buffer of $0.2 \%$ milk (Sigma, UK), 5\% fetal bovine serum (Gibco, Life Technologies, UK) and $0.1 \%$ Triton X-100 in PBS for $1 \mathrm{~h}$ at room temperature. Slides were then incubated with 53BP1 antibody (Novus Biologicals, CO, USA) at a dilution of 1:5000 in blocking buffer for $1 \mathrm{~h}$ at room temperature. They were then rinsed three-times with
PBS before being incubated with Alexafluor 488 Goat anti Rabbit secondary antibody (Invitrogen Molecular Probes, Oregon, USA) at a dilution of 1:1000 in blocking buffer and costained with ProLong ${ }^{\circledR}$ Gold Antifade mounting medium with DAPI. DNA damage was quantified by immunofluorescence detection of 53BP1 and nuclear foci were manually scored for 50 cells per slide using a Zeiss Axiovert $200 \mathrm{M}$ microscope (Carl Zeiss MicroImaging, LLC, USA).

\section{Au@DTDTPA induced ROS yields measured by DCFDA}

A total of $1.5 \times 10^{4}$ cells were plated and left to adhere for $24 \mathrm{~h}$ before being washed twice in PBS and supplemented with fresh medium containing $5 \mu \mathrm{M}$ DCFDA (Abcam, UK) for $40 \mathrm{~min}$. Media containing the dye was removed and replaced with fresh media containing $250 \mu \mathrm{g} / \mathrm{ml}$ AuNPs for varying time intervals. Samples were read on a fluorescent plate reader at excitation/ emission $495 / 529 \mathrm{~nm}$. A total of $5 \mu \mathrm{M}$ tert-butyl hydrogen peroxide was used as a positive control to demonstrate induction of ROS.

\section{Cone beam CT imaging \& in vivo radiation}

procedures using the small animal radiotherapy research platform

Cone beam CT (CBCT) images of a phantom loaded with different concentrations of Au@DTDTPA nanoparticles were obtained at $50 \mathrm{kV}$ and $0.6 \mathrm{~mA}$ using a small animal radiotherapy research platform (SARRP; XStrahl, Life Sciences). Analysis of CBCT datasets was performed by determining the CT numbers from the nanoparticle samples relative to water for defined regions of interest. A calibration curve for CT number relative to water as a function of AuNP concentration was obtained and used to correlate the in-phantom x-ray absorbance measurements with the pretreatment CBCT images. CBCT phantom experiments allowed the determination of intratumoral AuNP concentration by comparing the CT number ratio relative to tissue. For the experiments presented, CBCT data at $10 \mathrm{~min}$ post injection were analyzed and compared against in-phantom measurements.

Prior to CBCT-guided irradiation, mice were anesthetized by intraperitoneal injection of xylazine/ketamine. Au@DTDTPA nanoparticles were delivered by intratumoral injection into tumor bearing mice at a concentration of $8 \mathrm{mg} / \mathrm{kg}$ equating to $160 \mu \mathrm{g}$ of Au@DTDTPA per $20 \mathrm{~g}$ animal. Treatment planning was performed in MuriPlan (Xstrahl Life Sciences) to achieve uniform dose distributions across the tumor target by delivering two parallel opposed $10 \times 10 \mathrm{~mm}$ beams and avoiding critical structures. Therapeutic radiation doses were delivered 10-15 min after Au@ 
DTDTPA injection and CT scan. Animals were placed in a recovery incubator after the treatment.

\section{In vivo tumor growth delay model}

Six- to 8-week-old male Fox Chase SCID (severe combined immunodeficient) mice (Charles River Laboratories, Oxford, UK) were used as a xenograft model for PC-3 cells. A total of $1.5 \times 10^{6}$ cells in $80 \mu \mathrm{l}$ of phosphate-buffered saline (PBS) were implanted intradermally on to the flank of animals under inhalant anesthesia. Tumors were allowed to grow to $100 \mathrm{~mm}^{3}$ before being randomly assigned to one of four experimental groups; Au@DTDTPA + IR, 4 Gy IR only, Au@DTDTPA only $(8 \mathrm{mg} / \mathrm{kg})$ and untreated controls.Au@DTDTPA was administered as described above. Upon recruitment to the study animal weights and tumor volume were measured three-times weekly. Tumor volume was determined in three orthogonal dimensions. Experimental endpoints for individual animals were defined by tumor volume exceeding $400 \mathrm{~mm}^{3}$ or animals losing in excess of $10 \%$ of their body weight at the start of the experiment. Animals received food and water ad libitum. All experimental procedures were carried out in accordance with United Kingdom Home office approved protocols for in vivo experimentation.

\section{Statistical analysis}

All results presented are the mean of at least three independent experiments \pm standard error mean (SEM). Plots were obtained using Prism version 5.01, with statistically significant differences calculated using a twotailed unpaired t-test with a p-value of $\leq 0.05$ considered significant. Statistical significance for in vivo KaplanMeier survival curves was calculated using the MantelCox test with $\mathrm{p} \leq 0.05$ considered significant. Figure annotations of statistical significance are represented as follows: ${ }^{*} \mathrm{p} \leq 0.05,{ }^{* *} \mathrm{p} \leq 0.01,{ }^{* * *} \mathrm{p} \leq 0.001$.

\section{Results}

Physical properties of Au@DTDTPA

nanoparticles \& determination of intracellular concentrations

The physical properties of Au@DTDTPA are summarized in Figure $1 \mathrm{~A}-\mathrm{C}$. DTDTPA molecules were conjugated to the surface of the core AuNP via two thiol groups leading to the formation of a stable multilayered ligand shell. The mean core AuNP size determined by TEM was shown to be $2.4 \pm 0.5 \mathrm{~nm}$, with DTDTPA conjugation increasing the hydrodynamic size to $5.37 \pm 0.17 \mathrm{~nm}$. The synthesized particles had an overall negative surface charge of $-29.6 \mathrm{mV}$ at $\mathrm{pH}$ 7.4, exhibiting a highly homogenous distribution in solution with a PDI of $0.3 \pm 0.021$. Nonspecific protein absorption results in the formation of a protein corona that can negatively impact biological activity [31]. To establish the stability of Au@DTDTPA in serum containing medium nanoparticles were incubated in complete medium containing 10\% FBS. Particle size was determined at multiple time points over a $24 \mathrm{~h}$ period, with no significant change in hydrodynamic size or PDI indicating a high degree of stability in serum containing medium.

Intracellular concentrations of $\mathrm{Au}$ were calculated from a standard curve $\left(\mathrm{R}^{2}=0.992\right)$ of anticipated nanoparticle concentrations plotted against measured values using AAS (Figure 2A). Figure 2B-D shows the absolute quantification of intracellular Au@DTDTPA content determined by AAS in cells incubated with increasing concentrations of Au@DTDTPA particles for $24 \mathrm{~h}$. Two wash steps were used to ensure that only intracellular gold was quantified.

Cell line dependent variations were observed across all nanoparticle concentrations with a dose-dependent effect observed in PC3 and PNT2-C2 cells (Figure 2B \& D). PC3 cells exhibit minimal uptake at $100 \mu \mathrm{g} / \mathrm{ml}$, achieving mean intracellular Au concentrations of $0.29 \mathrm{pg} / \mathrm{cell}$, while DU145 and PNT2-C2 cells show a 2.23- and 3.79-fold increase, highlighting a cell type dependent variation in endocytosis. Increasing the Au@DTDTPA concentration from 100 to $250 \mu \mathrm{g} / \mathrm{ml}$ significantly increased the intracellular nanoparticle concentration by 5.4-, 1.17- and 2.05fold in PC3, DU145 and PNT2-C2 cells respectively following a $24 \mathrm{~h}$ exposure. Furthermore, doubling Au@DTDTPA concentrations to $500 \mu \mathrm{g} / \mathrm{ml}$ resulted in maximal intracellular concentrations of $3.65,1.8$ and $3.46 \mathrm{pg} / \mathrm{cell}$ in PC3, DU145 and PNT2-C2 cells, respectively, representing a close to linear uptake in PC3 and DU145 cells at higher concentrations. Au@DTDTPA concentrations exceeding $500 \mu \mathrm{g} / \mathrm{ml}$ were not investigated due to evidence of cytotoxicity at higher concentrations.

\section{Au@DTDTPA cytotoxicity}

The Alamar blue assay was used to test the shortterm cytotoxicity of Au@DTDTPA over a 28 h time period following initial exposure to Au@DTDTPA (24 h Au@DTDTPA and 4 h Alamar blue exposure). Exposure concentrations were the same as those used to determine intracellular uptake. Figure 3 represents cytotoxicity normalized to the mean viability of the control cells which were not exposed to AuNPs. Treatment with Au@DTDTPA negatively impacted shortterm viability, resulting in a mean reduction by $21.6 \%$ $\pm 1.59 \%$ in all cells treated with nanoparticles up to concentrations of $250 \mu \mathrm{g} / \mathrm{ml}$. Increasing the exposure concentration of Au@DTDTPA to $500 \mu \mathrm{g} / \mathrm{ml}$ resulted 
(A)

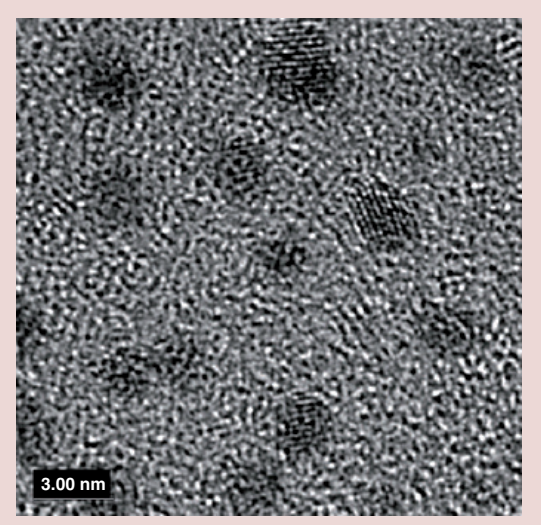

(B)

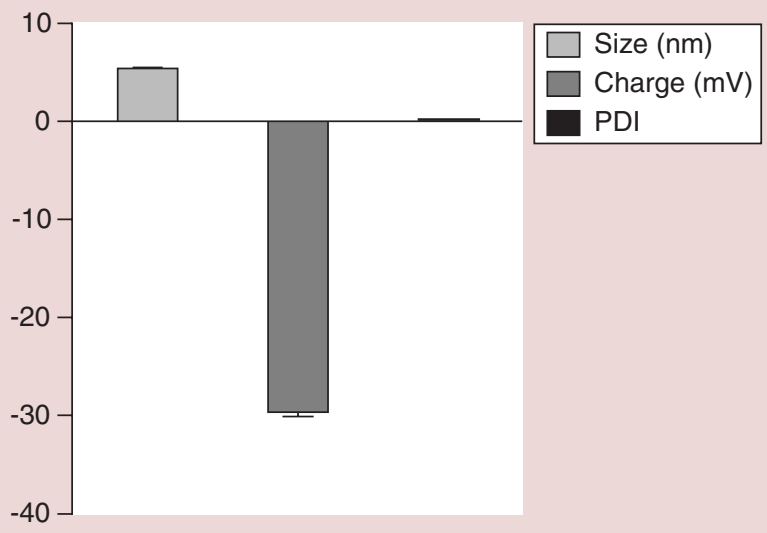

(C)

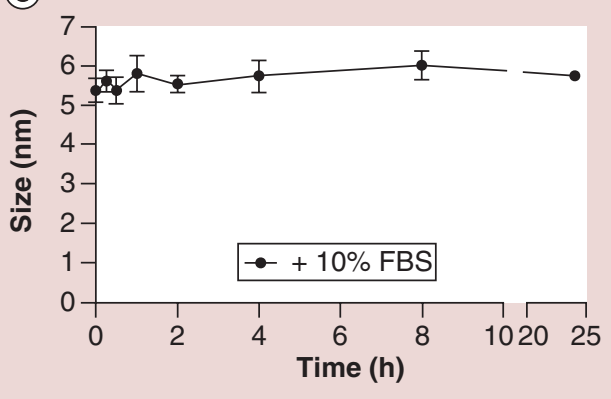

Figure 1. Physical characterization and stability of Au@DTDTPA nanoparticles. (A) High resolution transmission electron micrograph of Au@DTDTPA nanoparticles. (B) Dynamic light scattering analysis of Au@DTDTPA hydrodynamic size, charge and polydispersity index. (C) Au@DTDTPA hydrodynamic size following incubation in complete RPMI medium containing $10 \%$ FBS. Error bars represent SEM.

in significant $(\mathrm{p}<0.01)$ cytotoxicity reducing viability by up to $45.5 \%$ in DU145 cells, with equivalent high toxicity levels observed in both of the other cell lines.

\section{In vitro radiosensitization \& DNA damage measurements}

Long-term cytotoxicity was quantified by the gold standard colony formation assay using an intermediate exposure concentration of Au@DTDTPA at $250 \mu \mathrm{g} / \mathrm{ml}$ (Figure 4A). No significant reduction in clonogenicity was observed in any cell line over 12 days indicating that any increased reduction in surviving fraction when used in combination with radiation was attributable to a synergistic radiosensitizing effect and independent of any short-term toxicity measured using the Alamar Blue viability assay. Radiosensitization was determined following pretreatment of cells with various concentrations of Au@DTDTPA over $24 \mathrm{~h}$ then irradiating at a dose of 4 Gy. Figure 4B-D shows the survival fraction of Au@DTDTPA doped cells relative to IR alone. Pretreatment with $250 \mu \mathrm{g} / \mathrm{ml}$ of Au@DTDTPA resulted in an additional 84,68 and $87 \%$ reduction in colony formation relative to 4 Gy in PC3 DU145 and PNT2-
C2 cells, respectively. Further decreases in cell survival were observed when combining $500 \mu \mathrm{g} / \mathrm{ml} \mathrm{Au@}$ DTDTPA with a 4 Gy radiation dose, however, this concentration of nanoparticles was also associated with significant cytotoxicity (Figure 3). Consequently, all subsequent in vitro experiments were performed using a nanoparticle concentration of $250 \mu \mathrm{g} / \mathrm{ml}$.

DNA damage was quantified using the 53BP1 assay following a $24 \mathrm{~h}$ incubation with Au@DTDTPA prior to irradiation. Figure $5 \mathrm{~A}-\mathrm{C}$ shows the $53 \mathrm{BP} 1$ foci (a surrogate marker for DNA double strand breaks [DSB]) 1 and $24 \mathrm{~h}$ post irradiation, in the presence and absence of AuNPs. No significant difference in the level of DNA DSB damage were observed in the presence of Au@DTDTPA $1 \mathrm{~h}$ post radiation, indicating that damage induction is dominated by the effects of ionizing radiation (Figure $5 \mathrm{Ai}-\mathrm{Ci}$ ). Levels of residual/ unrepaired DSB were also quantified $24 \mathrm{~h}$ post radiation treatment where PC3 cells were shown to have 1.7-fold higher level of residual damage in the presence of Au@DTDTPA compared with cells receiving radiation only. These findings suggest that AuNPs have a negative impact DNA damage repair causing 
(A)

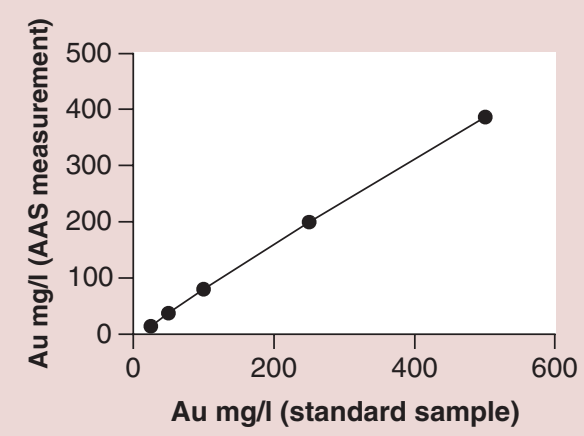

(C)

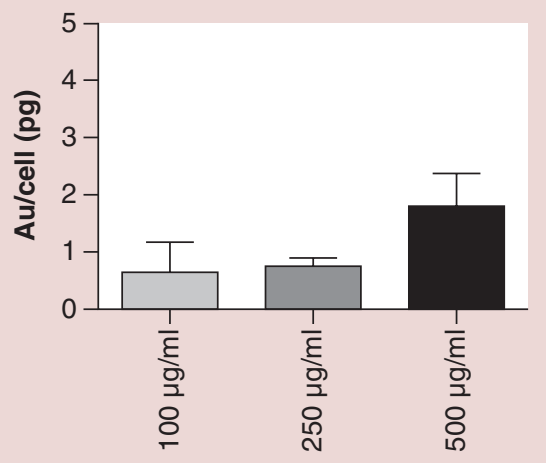

(B)

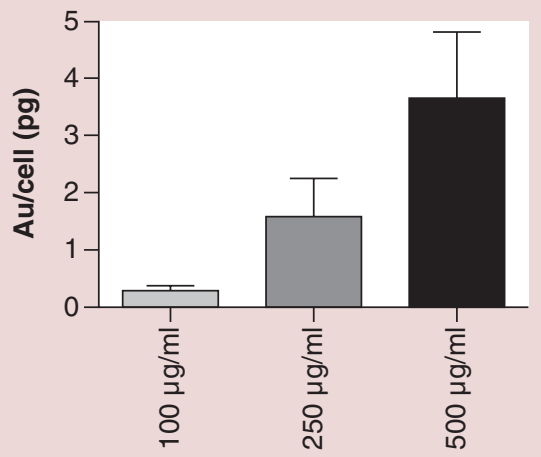

(D)

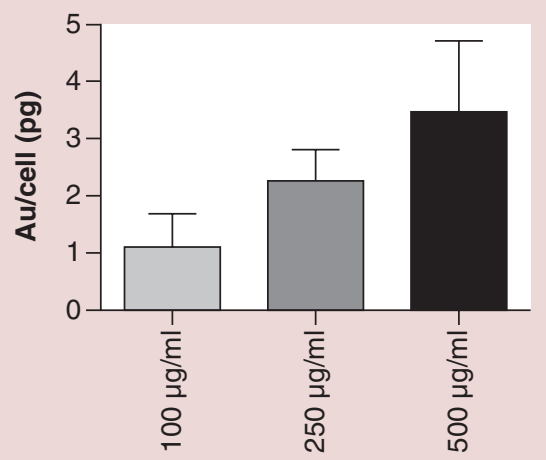

Figure 2. (A) Standard curve of Au@DTDTPA nanoparticles to determine intracellular Au concentrations using atomic absorption spectroscopy. (B-D) Intracellular concentration of Au determined by AAS following a $24 \mathrm{~h}$ incubation with increasing concentrations $(100,250$ and $500 \mu \mathrm{g} / \mathrm{ml})$ of Au@DTDTPA in PC3, DU145 and PNT2-C2 cells, respectively.

more persistent damage that may be due to more complex damage lesions in the nuclear DNA. A nonsignificant effect was observed in PNT2-C2 cells, the opposite effect was seen in DU145 cells, significantly $(\mathrm{p}<0.01)$ stimulating the damage response. The contribution of nanoparticle induced ROS was also deter- mined using DCFDA, a nonfluorescent compound that become fluorescent following oxidation by nonspecific ROS (Figure 5A.II-C.II). In all three cell lines, Au@DTDTPA resulted in a small time dependent increase in ROS yields, although not to a statistically level when compared against the untreated control
(A)

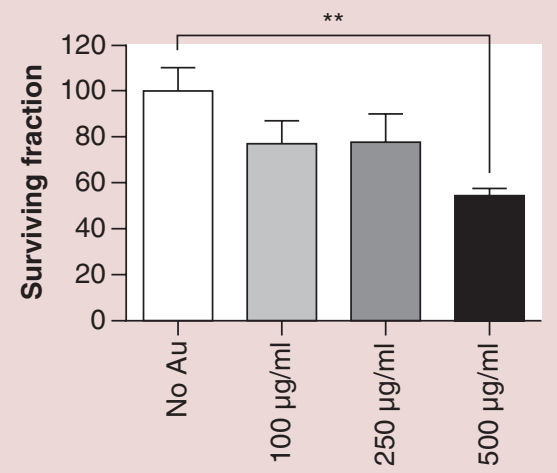

(B)

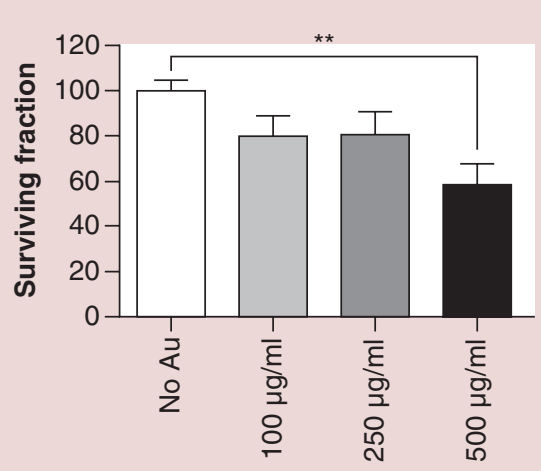

(C)

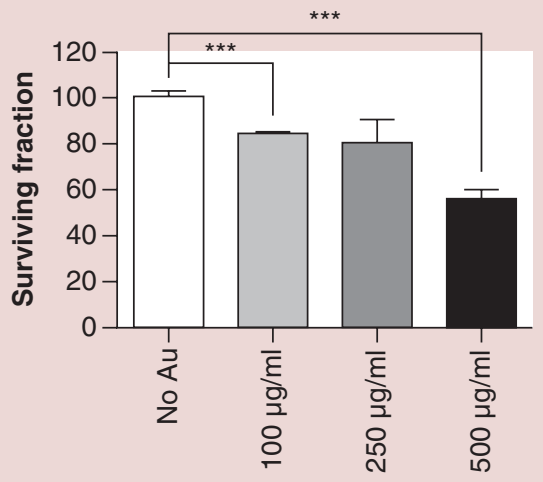

Figure 3. (A-C) PC3, DU145 and PNT2-C2 cytotoxicity data determined by fluorescent Alamar blue assay, following a $24 \mathrm{~h}$ incubation with Au@DTDTPA. Survival fraction was determined relative to the untreated control, while error bars represent SEM. 


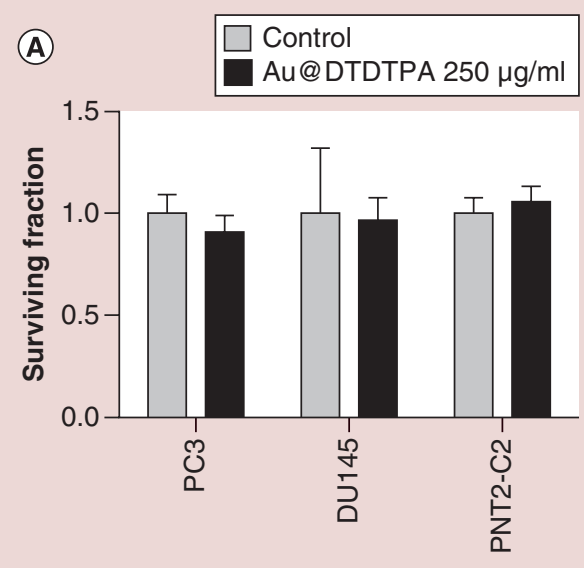

(C)

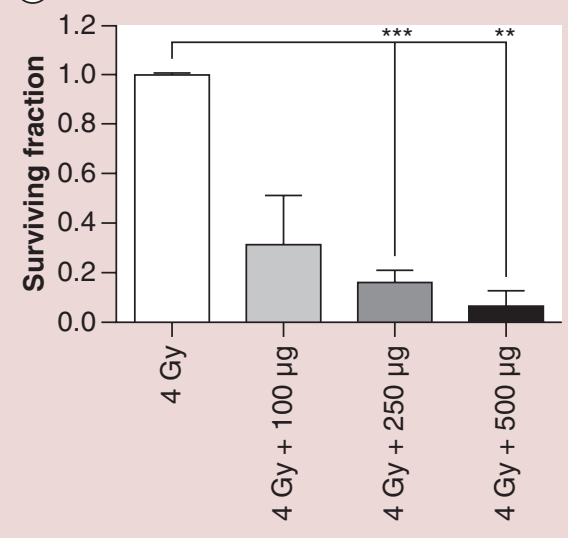

(B)

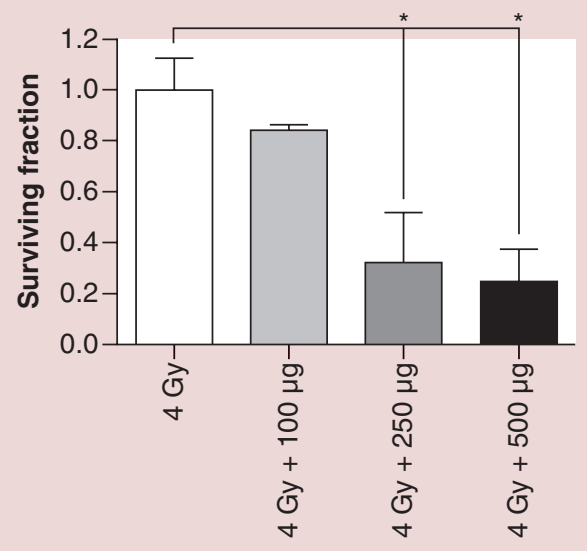

(D)

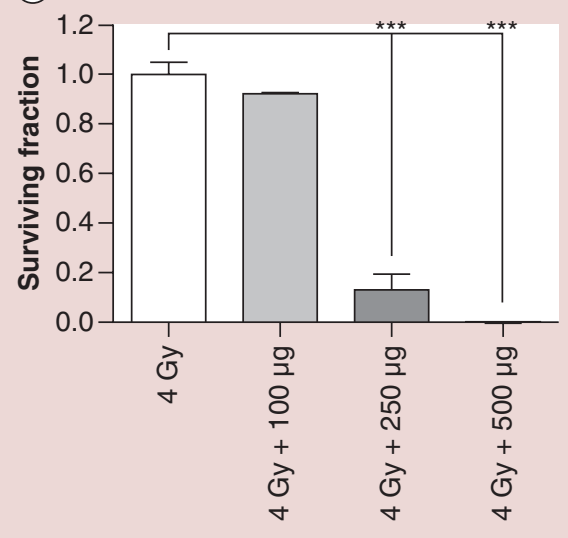

Figure 4. (A) Relative colony forming potential of PC3, DU145 and PNT2-C2 cells exposed to $250 \mu \mathrm{g} / \mathrm{ml}$ Au@ DTDTPA. Cells were exposed to Au@DTDTPA for $24 \mathrm{~h}$ prior to receiving a 4 Gy dose of $225 \mathrm{kVp}$ x-rays. (B-D) PC3, DU145 and PNT2-C2 radiosensitizing efficacy was determined by changes in colony forming efficacy relative to cells receiving radiation only.

cells. As a consequence the variable cell line dependent response, particularly with respect to DNA damage, and due to the significant in vitro radiosensitization, PC3 cells were selected to establish both the diagnostic and therapeutic potential of Au@DTDTPA in vivo.

\section{Enhancement of CBCT imaging}

Prior to in vivo imaging studies, $\mathrm{x}$-ray absorption experiments were carried out with nanoparticle solutions at various concentrations of Au@DTDTPA nanoparticles. CBCT images of phantoms containing $\mathrm{Au} @ D T D T P A$ at various gold concentrations (from 0.42 to $8.4 \mathrm{mg} / \mathrm{ml}$ ) were obtained at $50 \mathrm{kV}, 0.6 \mathrm{~mA}$ using the on-board CBCT imaging capabilities of the SARRP (Figure 6A). Au@DTDTPA nanoparticles show obvious contrast enhancement relative to water which increases linearly to plateau a concentrations above $4.2 \mathrm{mg} / \mathrm{ml}$ (Figure 6B).
In vivo images were then analyzed and the CT numbers relative to tissue were compared for the control group and AuNP injected group. Assuming the $10 \mathrm{~min}$ time-lapse post injection was suboptimal for tumor uptake, negative contrast was observed within the tumor interstitium. This setup was then used to study the differential contrast in the tumor or in AuNP accumulation around the tumor. Significant correlation was found between subcutaneous AuNP accumulation CT ratio and the in-phantom output for the $8.4 \mathrm{mg} /$ $\mathrm{ml}$ (Figure 6C). As anticipated, the tumor to tissue CT ratio showed no increase indicating minimal uptake in the tumor for the stated time point.

\section{Xenograft tumor growth delay model}

Fox Chase SCID mice implanted with intradermal PC3 tumors were weighed (an indicator of animal health) and tumor volume recorded three times weekly 

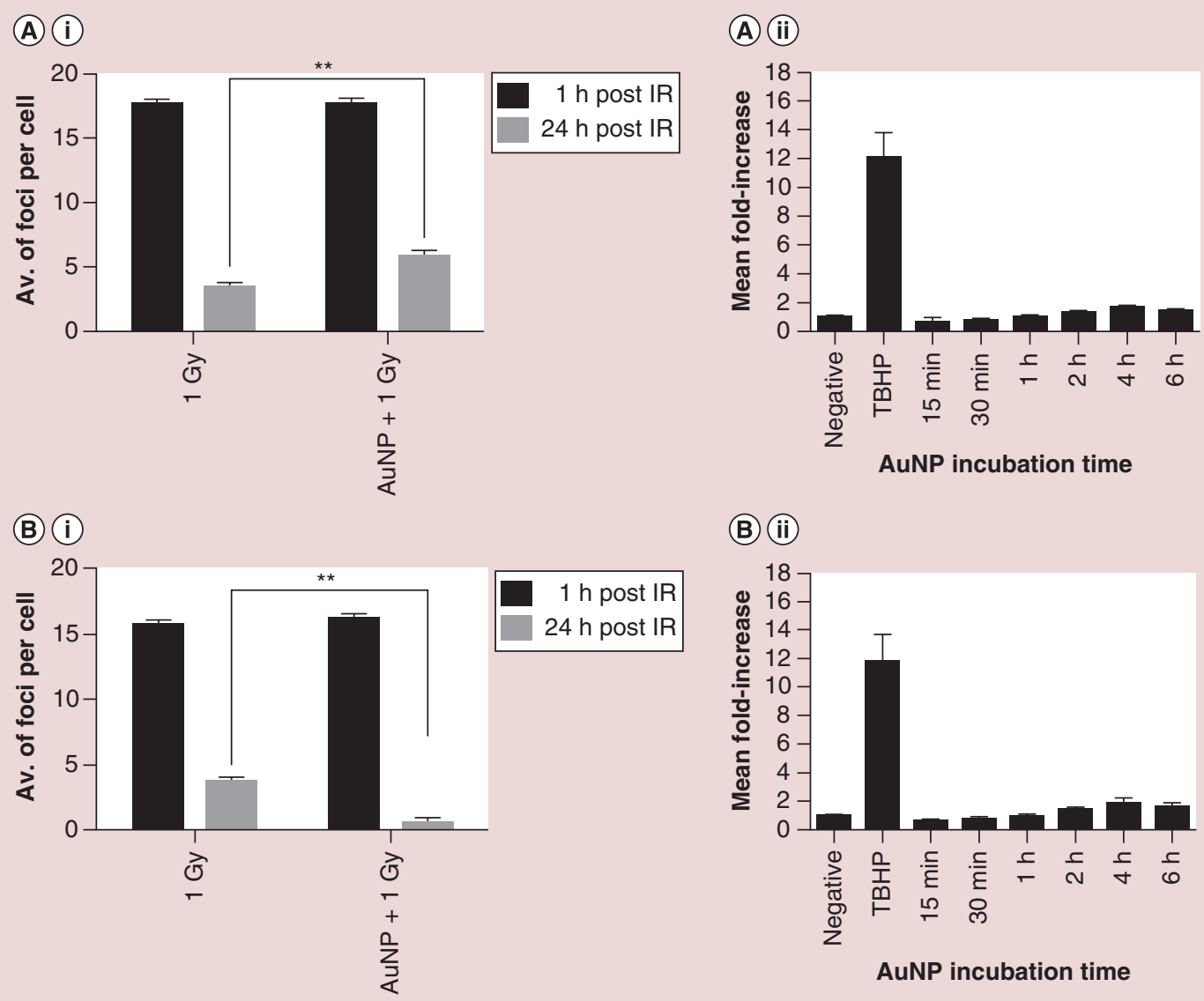

(B) (ii)

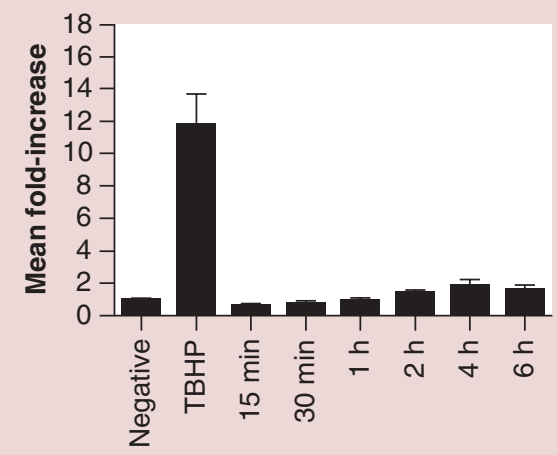

AuNP incubation time

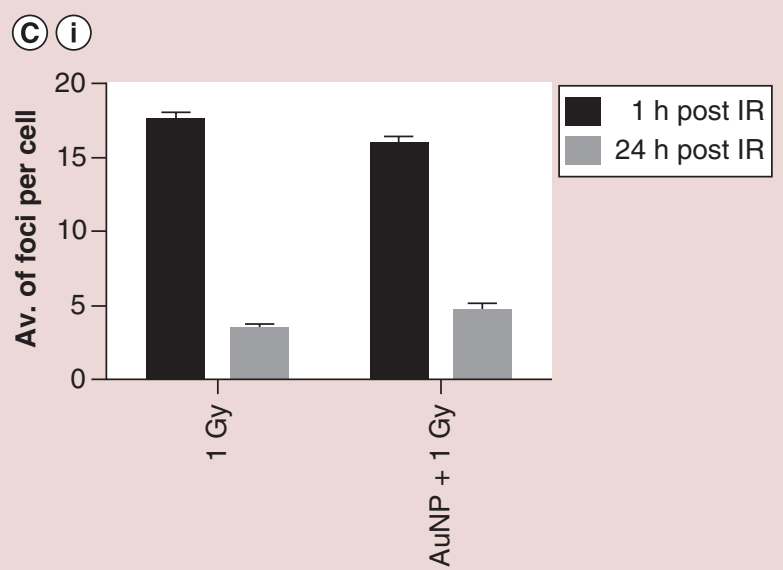

(C) (ii)

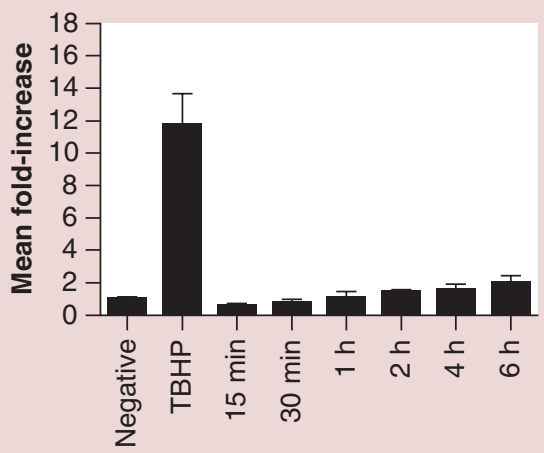

AuNP incubation time

Figure 5. DNA damage and reactive oxygen species yields following Au@DTDTPA treatment. DNA damage measurements were determined by immuno-fluorescent staining of 53BP1 following fixation 1 and $24 \mathrm{~h}$ post IR treatment. PC3 (5Ai) DU145 (5Bi) and PNT2-C2 (5Ci) cells were treated in the absence and presence of Au@ DTDTPA. Au@DTDTPA $(250 \mu \mathrm{g} / \mathrm{ml})$ induced reactive oxygen species was determined using DCFDA in PC3 (5Aii), DU145 (5Bii) and PNT2-C2 (5Cii) cells.

(Figure 7). Established tumors were defined as reaching $100 \mathrm{~mm}^{3}$ at which point animals were randomly assigned to one of four treatment groups. Mean animal weights per treatment group show only minor fluctuations over the course of the study, while all animals tolerated nanoparticle treatments well with no appar- ent signs of morbidity based on body condition scoring (Figure 7A).

Untreated control animals developed highly proliferative tumors, exhibiting a mean increase of $15 \%$ tumor volume per day. As a consequence all untreated control animals exceeded the experimental endpoint of 
$400 \mathrm{~mm}^{3}$ within 10 days of recruitment. Despite Au@ DTDTPA-mediated cytotoxicity observed in vitro, treatment alone at $8 \mathrm{mg} / \mathrm{kg}$ had a minimal impact on tumor growth extending survival by $2.6 \pm 1.4$ days. The impact of a single fraction of 4 Gy x-ray significantly ( $p$ $=0.008$ compared with untreated controls) impaired tumor growth resulting in tumor stasis for up to 16 days post treatment, after which progression was observed at an equivalent rate to that seen in Au@DTDTPA-treated animals. Consequently, radiation only treated animals took an additional $16.9 \pm 4.4$ days to reach $300 \mathrm{~mm}^{3}$ compared with control animals (Figure 7C). However, in agreement with the significant radiosensitizing effects observed in vitro, the combination of Au@DTDTPA and IR significantly $(\mathrm{p}=0.007)$ attenuated tumor growth over radiation alone, taking $38.34 \pm 1.9$ days for tumors to triple in volume, an extension in tumor growth delay of over 11 days. Both IR only and Au@DTDTPA
+ IR treated animals produced similar tumor growth characteristics, with a prolonged delay without tumor regression post treatment that was eventually overcome resulting in rapidly growing tumors.

Kaplan-Meier survival curves (Figure 7D) demonstrate a marked improvement in survival fraction for Au@DTDTPA + IR, both in terms of the first death (32 days compared with 18 days with IR alone) and last (42 vs 38 days). This is further reflected through median survival times of 38 days for Au@DTDTPA + IR compared with 29 days with IR alone, demonstrating a $31 \%$ increase in median survival with the combined treatment.

\section{Discussion}

The synthesis procedure for Au@DTDTPA nanoparticles yields a homogenous population with a hydrodynamic size of $5.37 \mathrm{~nm}$ and an overall negative sur-
(A)

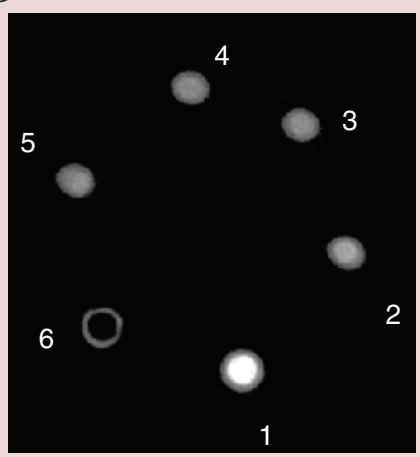

(C)

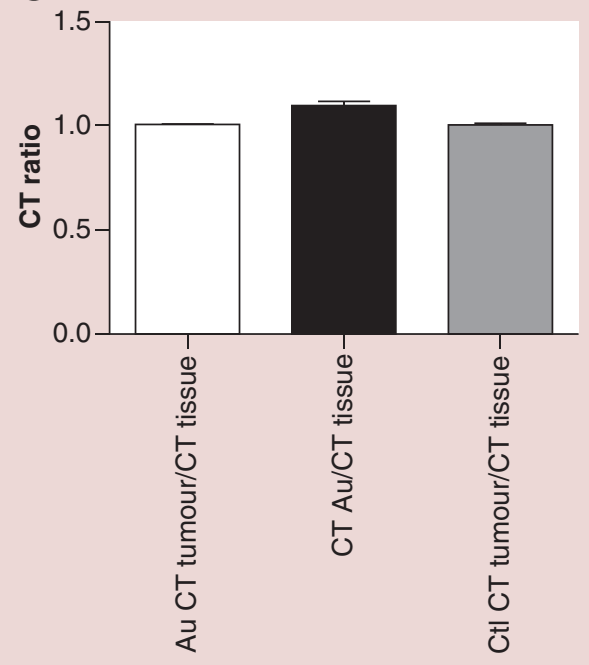

(B)

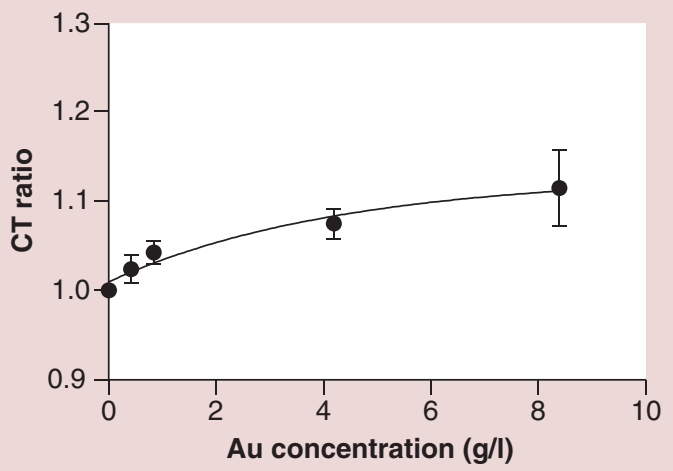

(D)
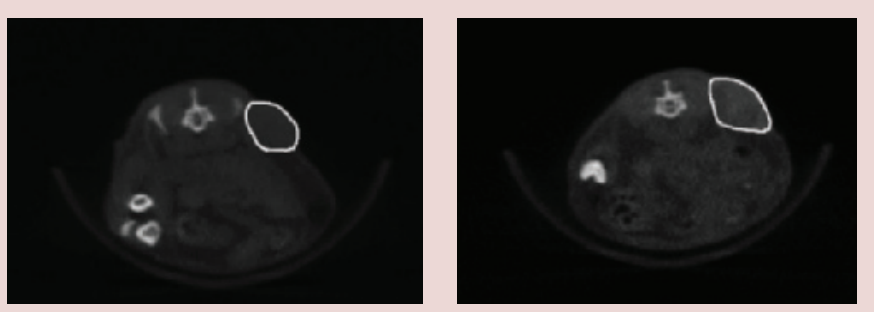

Figure 6. Phantom and in vivo cone beam CT imaging of Au@DTDTPA nanoparticles. (A) CBCT images for nanoparticles concentrations of 8.4 (1), 4.2 (2), 0.84 (3), 0.42 (4) mg/ml, water (5) and air (6). (B) Calibration curve of CBCT ratio (AuNP:water) for different nanoparticle concentrations. (C) CBCT enhancement values. (D) representative CBCT images (i) before, and (ii) after intratumoral injection with AuNPs. 
(A)

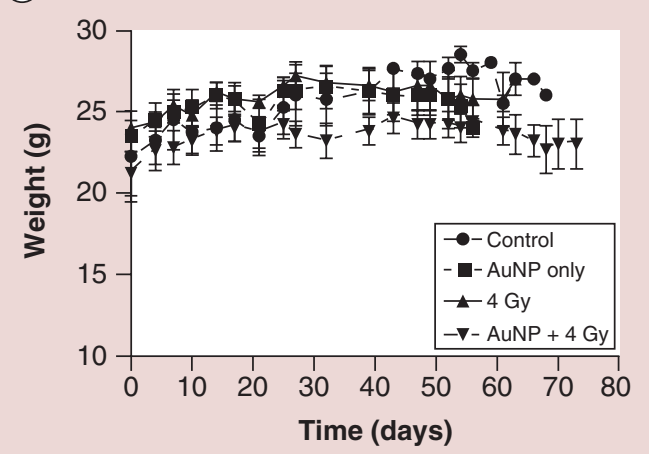

(C)

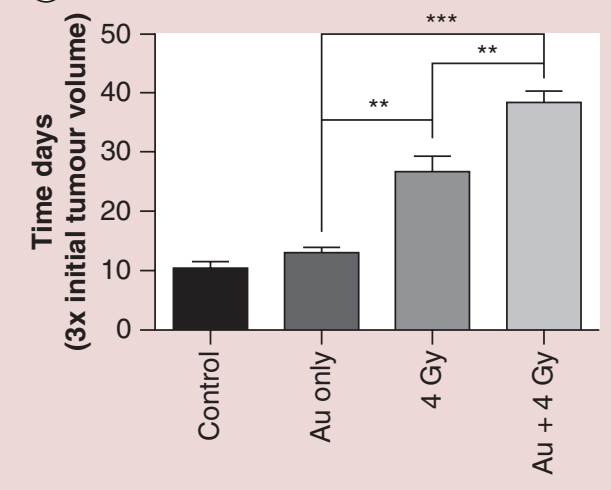

(B)

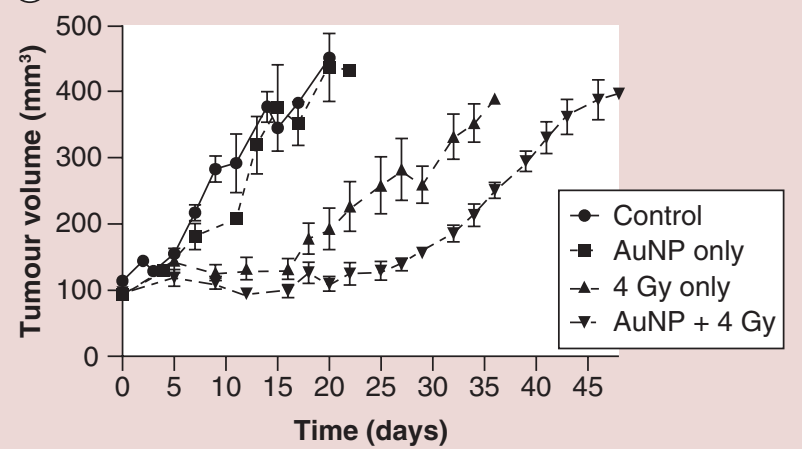

(D)

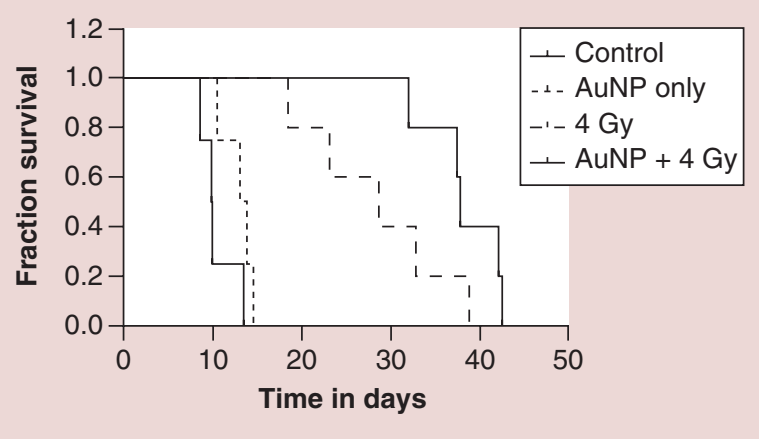

Figure 7. Xenograft tumor growth delay model in male Fox Chase severe combined immunodeficient mice implanted with PC3 prostate cancer cells. Animals were divided into experimental groups untreated, Au only (a single intratumoral injection at a concentration of $5 \mathrm{mg} / \mathrm{kg}$ Au@DTDTPA), $4 \mathrm{~Gy}$ and Au + $4 \mathrm{~Gy}$. IR was delivered as 4 Gy dose in two parallel opposed beams, guided by $\mathrm{CT}$ imaging on the small animal radiation research platform (SARRP). (A) Mean animal weight per treatment group indicating no weight loss. (B) Mean tumor growth characteristics of the four treatment groups. Tumors were permitted to grow until the predetermined experimental end point of $400 \mathrm{~mm}^{3}$. (C) Treatment efficacy defined by the delay in time taken for tumors to increase three-fold from the point of recruitment to the study. Statistical analysis was performed using a two-tailed t-test. (D) Kaplan-Meier survival curves demonstrating individual animal survival characteristics. Experimental start point varies from tumor implantation (A) to a tumor volume of $100 \mathrm{~mm}^{3}$ (B \& D).

face charge $(-29 \mathrm{mV})$. Incubation of nanoparticles in medium containing serum is a recognized methodology for assessing the evolution of a nonspecific protein corona [32]. MS analysis of medium containing serum identified more than 10,500 individual proteins ranging in concentrations up to $0.07 \mathrm{~g} / \mathrm{ml}$ [33]. Nanoparticles grafted with cationic surface groups generate positively charged particles that are designed to enhance electrostatic interactions with negatively charged cell membranes, thereby promoting nanoparticle internalization. However, this strategy is potentially flawed as it results in nonspecific interactions with serum proteins that coat the nanoparticle surface, influencing biological activity and clearance [34].

The negative $\zeta$ potential of Au@DTDTPA provides dual protection through electrostatic repulsion by preventing nanoparticle agglomeration and the absorption of serum proteins as evidenced by a stable hydro- dynamic size and polydispersity index over a 24-h period. Furthermore, the potent stabilizing properties of DTDTPA further validates the findings of Debouttière et al. (2006) who reported excellent stability of a DTDTPA gadolinium:Au conjugate across a broad spectrum ( $\mathrm{pH} 2-12)$ of $\mathrm{pH}$ solutions [23].

Conversely, electrostatic repulsion may negatively impact nanoparticle internalization. AAS was used to establish the concentration dependent effect of Au@DTDTPA on nanoparticle endocytosis. Nanoparticle uptake was observed in all cell lines reaching a maximum internal concentration of $3.65 \mathrm{pg} / \mathrm{cell}$, indicating that internalization occurred independent of surface charge. While a positive charged nanoparticle may initially result in higher uptake, the benefit of DTDTPA conjugation ensures that following in vivo administration nanoparticles are protected from opsonization and reticulo-endothelial clearance [35]. 
Cytotoxicity is a significant issue associated with emerging nanoparticle theranostic agents. While concerns exist with respect to gadolinium chelates used in MRI, AuNPs are generally considered to be biocompatible [36,37]. However, in our initial cell viability assays, all concentrations of Au@DTDTPA negatively impacted cell survival by approximately $20 \%$, while at the highest concentrations survival was reduced by up to $45 \%$. As a consequence, subsequent experiments were performed using the intermediate exposure concentration of $250 \mu \mathrm{g} /$ $\mathrm{ml}$. While this may appear as a limiting factor, it does not mirror the effects observed on long-term survival in vitro determined by clonogenic assay, nor were any adverse effects observed following in vivo administration.

In the context of clinical cancer treatments (cytotoxic chemotherapy or targeted agents) no agents are nontoxic and in some cases have significant toxicity indications. Taxane-based chemotherapies represent the first line of treatment for patients with metastatic prostate cancer [38]. Despite high-level sensitivity of prostate cancer cells (DU145) to docetaxel $\left(\mathrm{IC}_{50} 8.7\right.$ $\mathrm{nM}$ ), it is associated with significant toxicity issues in the clinic particularly in patients with hematopoietic abnormalities resulting in febrile neutropenia and acute hepatocellular toxicity [39].

To establish the radiosensitizing potential of $\mathrm{Au} @$ DTDTPA, PC3, DU145 and PNT2-C2 cells were exposed to various doses of nanoparticles prior to irradiation with a single $4 \mathrm{~Gy}$ dose. In most cases nanoparticle concentration plus radiation inversely correlated with survival, resulting in significant radiosensitizing effects (Figure 4). Importantly, these effects translated into highly significant PC3 tumor growth delay in animals treated with Au@DTDTPA, extending survival by $31 \%$ over animals receiving radiation only. The significance of this finding is increased when considered alongside existing simulated and experimental datasets. Based on Monte Carlo simulations of AuNP radiosensitization, intratumoral concentrations of almost $1 \%$ wt/wt (7 mg Au/g tumor) are required to achieve a twofold dose enhancement using $140 \mathrm{kVp}$ x-rays [40]. Similarly, early in vivo experimental studies by Hainfeld achieved substantial improvements in survival for animals treated with AuNPs in combination with radiation in both mammary carcinoma and glioblastoma tumor models [41,42]. However, translation of this preparation into a clinically relevant nanoparticle is hampered by high treatment concentrations $>1.35$ $\mathrm{mg} / \mathrm{g}$ body weight, which if used in the same manner for human subjects would equate to more than $100 \mathrm{~g}$ AuNP per patient for an $80 \mathrm{~kg}$ individual. In the current study, PC3 xenograft tumors were directly injected with a single treatment containing approximately 160 $\mu \mathrm{g}$ of $\mathrm{Au}$ prior to radiation treatment, representing clinically feasible concentrations of Au per treatment. Although, direct intratumoral injections have limited applicability in the metastatic context, our approach could hold significant potential for a specific cohort of prostate cancer patients with locally advanced disease that has progressed beyond the prostatic capsule with possible invasion into the pericapsular tissue and seminal vesicles. Approximately $30 \%$ of T3-T4 lymph node negative, metastasis negative prostate cancers ultimately fail treatment [43]. Intervention with radical radiotherapy in combination with effective radiosensitizers such as Au@DTDTPA represents an alternative, viable solution to improving treatment outcome for these patients. The application of a potent radiosensitizer used in combination with a lower radiation treatment dose could significantly minimizing off-target toxicity to tissues such as the rectum and bladder. Additionally, enhanced CT-contrast afforded by the Au@DTDTPA (Figure 6) could be incorporated into cone beam-CT image-guided radiotherapy treatment plans to further minimize the risk of off-target damage.

\section{Conclusion}

Used in the appropriate clinical context, as described above, or optimized with specific targeting motifs to ensure efficient tumor specific accumulation, AuNPs hold significant potential as theranostic agents for radiotherapy. In the current study, surface conjugation with DTDTPA provided stable protection against nanoparticle agglomeration and protein absorption, while retaining an acceptable endocytotic uptake to yield potent radiosensitization in both in vitro and in vivo models. Further optimization of nanoparticle dosing schedule with fractionated radiation protocols will help to facilitate transition from preclinical study to first in man trials.

\section{Financial \& competing interests disclosure}

The authors are grateful to the European Commission Framework 7 Programme (grant number EC FP7 MC - ITN - 608163 - ARGENT) for funding their work. The authors have no other relevant affiliations or financial involvement with any organization or entity with a financial interest in or financial conflict with the subject matter or materials discussed in the manuscript apart from those disclosed.

No writing assistance was utilized in the production of this manuscript.

\section{Ethical conduct of research}

The authors state that they have obtained appropriate institutional review board approval or have followed the principles outlined in the Declaration of Helsinki for all human or animal experimental investigations. In addition, for investigations involving human subjects, informed consent has been obtained from the participants involved. 
Open access

This work is licensed under the Creative Commons Attribution-NonCommercial 4.0 Unported License. To view a copy of this license, visit http://creativecommons.org/licenses/bync-nd/4.0/

\section{Executive summary}

- Dithiolated diethylenetriamine pentaacetic acid (DTDTPA) functionalized gold nanoparticles (AuNPs) were successfully synthesized and characterized. Furthermore, $5 \mathrm{~nm}$ AuNPs remained stable at $1 \mathrm{M} \mathrm{NaCL}$ for a $24 \mathrm{~h}$ period.

- Au@DTDTPA proved nonsignificantly toxic up to $500 \mu \mathrm{g} / \mathrm{ml}$ in the short term, while $250 \mu \mathrm{g} / \mathrm{ml} \mathrm{had} \mathrm{no} \mathrm{impact}$ on cell survival after 14 days.

- Atomic absorption spectroscopy demonstrated a concentration-dependent uptake of AuNPs in prostate cells, with significant uptake at nontoxic gold concentrations.

- Significant radiosensitization was achieved in all three prostate cell lines following exposure to $250 \mu \mathrm{g} / \mathrm{ml} \mathrm{Au} @$ DTDTPA. PC3 cells showed the greatest enhancement at $87 \%$ compared with radiation alone at $4 \mathrm{~Gy}$.

- DNA lesions and reactive oxygen species production were quantified $24 \mathrm{~h}$ post radiation, demonstrating a 1.7fold increase in DNA double strand breaks, following incubation with Au@DTDTPA in PC3 cells. No significant reactive oxygen species elevation was detected over a 6 - $\mathrm{h}$ period.

- A xenograft tumor delay model was used to determine the therapeutic and diagnostic capabilities of Au@ DTDTPA in vivo, with a CT image enhancement of $10 \%$ observed.

- Furthermore, when combined with $4 \mathrm{~Gy}+$ Au@DTDTPA survival time increased by $29 \%$ from $16.9 \pm 4.4$ days to $38.3 \pm 1.9$ days compared with animals receiving radiation only, demonstrating significant efficacy.

- This study includes one of the first examples of successful in vivo radiosensitization in prostate cancer, which is further complimented by CT enhancement and extensive in vitro experimentation.

\section{References}

Papers of special note have been highlighted as: $\bullet$ of interest; $\bullet$ of considerable interest

1 Jung $S$, Nam J, Hwang $S$ et al. Theragnostic pH-sensitive gold nanoparticles for the selective surface enhanced Raman scattering and photothermal cancer therapy. Anal. Chem. 85, 7674-7681 (2013).

2 Gobbo OL, Sjaastad K, Radomski MW, Volkov Y, PrinaMello A. Magnetic nanoparticles in cancer theranostics. Theranostics 5(11), 1249-1263 (2015).

3 Mukherjee P, Misra SK, Gryka MC et al. Tunable luminescent carbon nanospheres with well-defined nanoscale chemistry for synchronized imaging and therapy. Small 11(36), 4691-4703 (2015).

4 Perala SRK, Kumar S. On the mechanism of metal nanoparticle synthesis in the Brust-Schiffrin method. Langmuir 29(31), 9863-9873 (2013).

5 Balasubramanian SK, Yang L, Yung L-YL, Ong C-N, Ong W-Y, Yu LE. Characterization, purification, and stability of gold nanoparticles. Biomaterials 31(34), 9023-9030 (2010).

6 Wang Y, Chen LQ, Li YF, Zhao XJ, Peng L, Huang CZ. A one-pot strategy for biomimetic synthesis and self-assembly of gold nanoparticles. Nanotechnology 21(30), 305601 (2010).

7 Connor EE, Mwamuka J, Gole A, Murphy CJ, Wyatt MD. Gold nanoparticles are taken up by human cells but do not cause acute cytotoxicity. Small 1(3), 325-327 (2005).

8 Narayanan S, Sathy BN, Mony U, Koyakutty M, Nair SV, Menon D. Biocompatible magnetite/gold nanohybrid contrast agents via green chemistry for MRI and CT bioimaging. ACS Appl. Mater. Interfaces 4(1), 251-260 (2012).
9 Kunzmann A, Andersson B, Thurnherr T, Krug H, Scheynius A, Fadeel B. Toxicology of engineered nanomaterials: focus on biocompatibility, biodistribution and biodegradation. Biochim. Biophys. Acta 1810(3), 361-373 (2011).

10 Su G, Yang C, Zhu J-J. Fabrication of gold nanorods with tunable longitudinal surface plasmon resonance peaks by reductive dopamine. Langmuir 31(2), 817-823 (2015).

11 Yuan H, Khoury CG, Wilson CM, Grant GA, Bennett AJ, Vo-Dinh T. In vivo particle tracking and photothermal ablation using plasmon-resonant gold nanostars. Nanomed. Nanotechnol. Biol. Med. 8(8), 1355-1363 (2012)

12 Hainfeld JF, Slatkin DN, Focella TM, Smilowitz HM. Gold nanoparticles: a new x-ray contrast agent. Br. J. Radiol. 79, 248-253 (2006).

- The first and most comprehensive study of gold nanoparticles as a CT contrast agent.

13 Cole LE, Ross RD, Tilley JM, Vargo-Gogola T, Roeder RK. Gold nanoparticles as contrast agents in x-ray imaging and computed tomography. Nanomedicine 10(2), 321-341 (2015).

14 Kircher MF, de la Zerda A, Jokerst JV et al. A brain tumor molecular imaging strategy using a new triple-modality MRI-photoacoustic-Raman nanoparticle. Nat. Med. 18(5), 829-834 (2012).

15 Labouta HI, Kraus T, El-Khordagui LK, Schneider M. Combined multiphoton imaging-pixel analysis for semiquantitation of skin penetration of gold nanoparticles. Int. J. Pharm. 413(1-2), 279-282 (2011).

16 Luke GP, Yeager D, Emelianov SY. Biomedical applications of photoacoustic imaging with exogenous contrast agents. Ann. Biomed. Eng. 40 (2), 422-437 (2012). 
17 Butterworth KT, McMahon SJ, Currell FJ, Prise KM. Physical basis and biological mechanisms of gold nanoparticle radiosensitization. Nanoscale 4(16), 4830 (2012).

18 Misawa M, Takahashi J. Generation of reactive oxygen species induced by gold nanoparticles under x-ray and UV Irradiations. Nanomedicine 7(5), 604-614 (2011).

19 McMahon SJ, Hyland WB, Muir MF et al. Nanodosimetric effects of gold nanoparticles in megavoltage radiation therapy. Radiother. Oncol. 100(3), 412-416 (2011).

20 Reuveni T, Motiei M, Romman Z, Popovtzer A, Popovtzer R. Targeted gold nanoparticles enable molecular CT imaging of cancer: an in vivo study. Int. J. Nanomedicine 6, 2859-2864 (2011).

21 Matsumura Y, Maeda H. A new concept for macromolecular therapeutics in cancer chemotherapy: mechanism of tumoritropic accumulation of proteins and the antitumor agents smancs. Cancer Res. 46, 6387-6392 (1986).

22 Debouttière P-J, Roux S, Vocanson F et al. Design of gold nanoparticles for magnetic resonance imaging. Adv. Funct. Mater. 16(18), 2330-2339 (2006).

- $\quad$ First reportof Au@DTDTPA, including synthesis and characterization.

23 Alric C, Miladi I, Kryza D et al. The biodistribution of gold nanoparticles designed for renal clearance. Nanoscale 5(13), 5930-5939 (2013).

- Extensive in vivo biodistribution study of Au@DTDTPA, including organ accumulation and clearance.

24 Alric C, Taleb J, Le Duc G et al. Gadolinium chelate coated gold nanoparticles as contrast agents for both $\mathrm{x}$-ray computed tomography and magnetic resonance imaging. J. Am. Chem. Soc. 130 (18), 5908-5915 (2008).

- Use of Au@DTDTPA + gadolinium ions to enhance MRI.

25 Miladi I, Alric C, Dufort $S$ et al. The in vivo radiosensitizing effect of gold nanoparticles based MRI contrast agents. Small. 10(6), 1116-1124 (2014).

26 Wolfe T, Chatterjee D, Lee J et al. Targeted gold nanoparticles enhance sensitization of prostate tumors to megavoltage radiation therapy in vivo. Nanomedicine 11(5), 1277-1283 (2015).

- Only other known in vivo radiosensitization of prostate tumors using gold nanoparticles.

27 Brust M, Fink J, Bethell D, Schiffrin DJ, Kiely C. Synthesis and reactions of functionalised gold nanoparticles. J. Chem. Soc. Chem. Commun. 16, 1655 (1995).

28 Lang SH, Hyde C, Reid IN et al. Enhanced expression of vimentin in motile prostate cell lines and in poorly differentiated and metastatic prostate carcinoma. Prostate. 52, 253-263 (2002).

29 Puck T, Marcus P. ACTION of x-rays on mammalian cells. J. Exp. Med. 103(39), 653-666 (1956).
30 Jain S, Coulter JA, Hounsell AR et al. Cell-specific radiosensitization by gold nanoparticles at megavoltage radiation energies. Int. J. Radiat. Oncol. Biol. Phys. 79(2), 531-539 (2011).

31 Fleischer CC, Payne CK. Nanoparticle-cell interactions: molecular structure of the protein corona and cellular outcomes. Acc. Chem. Res. 47(8), 2651-2659 (2014).

32 Barran-Berdon AL, Pozzi D, Caracciolo G et al. Time evolution of nanoparticle - protein corona in human plasma: relevance for targeted drug delivery. Langmuir 29, 6485-6494 (2013).

33 Nanjappa V, Thomas JK, Marimuthu A et al. Plasma Proteome Database as a resource for proteomics research: 2014 update. Nucleic Acids Res. 42, D959-D965 (2014).

34 Cedervall T, Lynch I, Lindman S et al. Understanding the nanoparticle-protein corona using methods to quantify exchange rates and affinities of proteins for nanoparticles. Proc. Natl Acad. Sci. USA 104(7), 2050-2055 (2007).

35 Longmire M, Choyke P, Kobayashi H. Clearance properties of nano-sized particles and molecules as imaging agents: considerations and caveats. Nanomedicine 3(5), 703-717 (2008).

36 Shukla R, Bansal V, Chaudhary M, Basu A, Bhonde RR, Sastry M. Biocompatibility of gold nanoparticles and their endocytotic fate inside the cellular compartment: a microscopic overview. Langmuir 21(23), 10644-10654 (2005).

37 Chandran PR, Naseer M, Udupa N, Sandhyarani N. Size controlled synthesis of biocompatible gold nanoparticles and their activity in the oxidation of NADH. Nanotechnology 23(1), 015602 (2011).

38 Lam E, Flaig T. Upfront chemotherapy for metastatic prostate cancer. Oncology 29(12), pii: 214810 (2015).

39 Beer TM. Weekly high-dose calcitriol and docetaxel in metastatic androgen-independent prostate cancer. J. Clin. Oncol. 21(1), 123-128 (2003).

40 Cho SH. Estimation of tumour dose enhancement due to gold nanoparticles during typical radiation treatments: a preliminary Monte Carlo study. Phys. Med. Biol. 50(15), N163-N173 (2005).

41 Hainfeld J, Smilowitz H, O'Connor M, Dilmanian F, Slatkin D. Gold nanoparticle imaging and radiotherapy of brain tumors in mice. Nanomedicine 8(10), 1601-1609 (2012).

42 Hainfeld JF, Slatkin DN, Smilowitz HM. The use of gold nanoparticles to enhance radiotherapy in mice. Phys. Med. Biol. 49(18), 309-315 (2004).

- First significant report of gold nanoparticle radiosensitization.

43 Warde P, Mason M, Ding K et al. Combined androgen deprivation therapy and radiation therapy for locally advanced prostate cancer: a randomised, Phase 3 trial. Lancet 378(9809), 2104-2111 (2011). 